\title{
Intermédialités
}

Histoire et théorie des arts, des lettres et des techniques

Intermediality

History and Theory of the Arts, Literature and Technologies

\section{Disparaître à présent. Introduction}

\section{George Varsos et Valeria Wagner}

Numéro 10, automne 2007

Disparaître

Disappearing

URI : https://id.erudit.org/iderudit/1005549ar

DOI : https://doi.org/10.7202/1005549ar

Aller au sommaire du numéro

Éditeur(s)

Centre de recherche sur l'intermédialité

ISSN

1705-8546 (imprimé)

1920-3136 (numérique)

Découvrir la revue

Citer ce document

Varsos, G. \& Wagner, V. (2007). Disparaître à présent. Introduction.

Intermédialités / Intermediality, (10), 9-16. https://doi.org/10.7202/1005549ar 


\title{
Disparaître à présent Introduction
}

\author{
George Varsos \\ Valeria Wagner
}

U

ne disparition célèbre ouvre les histoires de la littérature occidentale:

celle d'Ulysse dans l'Odyssée. Nous nous reconnaîtrons, contemporains du $\mathrm{XxI}^{\mathrm{e}}$ siècle, dans la détresse que l'absence inexpliquée du héros suscite parmi ses proches. Au début du récit, Télémaque entreprend un voyage auprès des anciens combattants de la guerre de Troie pour les interroger à propos de son père, dont il n’a pas de nouvelles. Il est encouragé par la déesse Athéna qui arrive à Ithaque sous la forme d'un étranger, ami d'Ulysse, et qui essaye d'assurer Télémaque que son père est encore vivant et sera bientôt de retour suivant la volonté des dieux. Mais ce n'est pas de songer à la mort de son père qui chagrine tant Télémaque; plutôt, ce qui le désespère - le laisse sans perspective d'avenir c'est l'incertitude quant à son sort. C'est ainsi qu'il élabore une distinction nette entre la condition de mort et celle de disparu, qui prévaut jusqu'à nos jours (I, $236-243)$ :

en effet, je ne serais pas si triste de sa mort

si du moins il était tombé parmi les gens à Troie,

ou dans les bras des siens, une fois la guerre achevée:

là les Panachéens lui eussent élevé un tertre

et à son fils, il eût encore légué sa haute gloire

Maintenant les Harpyes l'ont emporté sans gloire;

il est parti obscur, ignoré il ne m’a laissé

que les pleurs et les plaintes ${ }^{1}$;

En tant que disparu, Ulysse a été privé non pas tellement de sa vie comme de la raison d'être du héros homérique, de sa «gloire » (kleos) ou, plus concrètement, de sa réputation ou renommée, voire de la possibilité de raconter les faits saillants

1. Homère, L’Odyssée, trad. Philippe Jacottet, Paris, Éditions La Découverte et Syros, coll. «La Découverte/Poche», 2000, p. 20. 
de sa vie. Il est parti «obscur» (aïstos)et «ignoré » (apystos) regrette Télémaque, personne ne l'a vu ni ne saurait dire quelque chose de certain sur son départ ${ }^{2}$. Impossible, alors, de soulager la douleur en marquant l'accomplissement de sa vie et la certitude de sa mort en érigeant un tertre.

L'éminence élevée et bien visible du tombeau est étroitement liée, chez Homère, à la configuration des faits et des expériences qui composent une vie humaine accomplie, à la construction et à la transmission de leur récit raconté à la postérité, aux «hommes à venir ${ }^{3}$ ». La mort certaine devient ainsi une condition pour la formation de la mémoire des survivants. Saisissant ce lien entre mort, transmission d'expériences et narration, Walter Benjamin dira même au sujet du conteur traditionnel, que «la mort est la sanction de tout ce qu'il peut raconter » et que «c'est de la mort qu'il tient son autorité4». C'est la présence de la figure du mourant dans la société traditionnelle qui attribuerait ainsi à la vie humaine la forme communicable qui soutient la vérité du récit de son histoire, et donc sa place dans la mémoire humaine:

Or c'est surtout chez le mourant que prend forme communicable non seulement le savoir ou la sagesse d'un homme, mais au premier chef la vie qu'il a vécue, c'est-àdire la matière dont sont faites les histoires. De même qu'au terme de son existence,

2. Aïstos est étymologiquement lié au verbe eido (proche du latin video) dont les formes multiples et très variées dénotent la faculté de la vue ou la capacité de voir, donc d'être témoin des faits et de savoir ou d'être bien informé à cet égard, mais aussi la capacité d'être vu et d'émerger ou d'apparaître ou de se présenter, ainsi que de ressembler ou de sembler ou de paraître. Historia qui implique, plus spécifiquement, connaissance à base de questions et de quête, est très probablement lié au même mot. Apystos a le sens de celui dont on n'entend pas parler ou à propos duquel on ne peut nullement se renseigner ou on ne sait comment enquêter.

3. Le tombeau peut être humble, tel que Elpénor, le compagnon disparu sur l'île de Circé, le demande à Ulysse, lorsque ce dernier rencontre son âme dans le pays des morts (XI, 75-78: L'Odyssée, p. 180):

dresse-moi un tombeau sur les rives de la mer grise

pour qu'il rappelle un malheureux aux hommes à venir.

Fais ces choses pour moi, sur mon tertre plante la rame avec quoi je ramais parmi mes compagnons, quand je vivais!

Le tombeau d'Achille, par contre, tel qu'Agamemnon le décrit dans la deuxième descente au pays des morts, est un monticule «sans défaut» dont la grandeur et situation permettent que «par la mer, il fût visible de très loin / aux hommes d'aujourd'hui et aux hommes de l'avenir» (XXIV, 8o-84: L'Odyssée, p. 382).

4. Walter Benjamin, «Le conteur. Réflexions sur l'œuvre de Nicolas Leskov» [1936], dans Euvres III, trad. Maurice de Gandillac, Rainer Rochlitz et Pierre Rusch, Paris. Éditions Gallimard, coll. «Folio essais», 2000, p. 130. 
il voit défiler intérieurement une série d'images - visions de sa propre personne, dans lesquelles, sans s'en rendre compte, il s'est lui-même rencontré - , ainsi, dans ses expressions et dans ses regards, surgit soudain l'inoubliable, qui confère à tout ce qui a touché cet homme l'autorité que revêt aux yeux des vivants qui l'entourent, à l'heure de sa mort, même le dernier des misérables. C'est cette autorité qui est à l'origine du récit5.

Rappelons que Benjamin distingue, d'une part, entre la mort en tant qu'événement présent dans la vie commune d'une société traditionnelle, qui déclenche le récit du conteur (représenté dans son argument par Nicolas Leskov) et, d'autre part, entre la mort exclue des lieux de la vie quotidienne bourgeoise, qui coïncide, dans le roman moderne, avec le terme de la narration, son dernier mot. Dans un cas, la mort donne du relief aux faits, tristes ou heureux, d'une vie accomplie, lesquels pourront être évoqués et reconfigurés par des récits multiples et variés. Dans l'autre, il n'y a qu'un seul récit d'autorité, qui se confond avec le déroulement et le sens global de la vie terminée et se ferme sur lui-même au terme de la narration. Dans un cas, donc, la mort ouvre sur des récits, dans l'autre, elle en est la clôture. Mais dans un cas comme dans l'autre, l'incertitude que génère la disparition entrave de manière distincte la possibilité même de donner forme à «la matière dont sont faites les histoires », comme dit Benjamin, affectant par là la possibilité de tout récit.

Plus proches de nos jours, les plaintes suscitées par la disparition des êtres chers expriment avec acuité l'aporie du récit quasiment impossible : "C'est quelque chose qui n'a pas de fin... il n'y pas de fin et c'est le plus douloureux, cela ne peut finir de... de clore, disons ", témoigne, par exemple, une femme dans un film de la réalisatrice argentine Albertina Carri - elle-même fille de parents disparus, et aux prises avec le paradoxe de vouloir maintenir la mémoire de leur disparition et de devoir continuer à vivre dans l'irrésolution qu'elle entraîne ${ }^{6}$. Ce paradoxe ne concerne pas seulement les proches de personnes disparues, et ne

5. Walter Benjamin, «Le conteur», p. 130.

6. «Es una cosa como que no tiene fin... no hay un final que eso es lo más doloroso, que no puede terminar de... de cerrar digamos.» Tiré de Los Rubios (Les blonds, 2003), Albertina Carri, Argentine. Ce témoignage porte sur les disparitions massives en Argentine pendant la dictature militaire de 1976-83. Mais des témoignages dans ce sens abondent, ces mots auraient pu être dits dans maintes autres contextes. Voir à ce sujet Alain Brossat et Jean-Louis Déotte (dirs.), L'époque de la disparition. Politique et esthétique, Paris, L'Harmattan, coll. «Esthétiques », 2000. Pour une discussion du film de Carri, voir Valeria Wagner, «Unhostly Historical Discourses in Ariel Dorfman’s Heading South, Looking North. A Bilingual Journey and Albertina Carri's film The Blonds », Discourse $27.2 \& 27.3$, spring and fall 2005 , p. $155^{-178}$. 
découle pas uniquement de la disparition de personnes. En effet, aujourd'hui la question de la disparition acquiert un poids d'autant plus paradigmatique que les technologies de la domination politique, de la guerre ou de la coercition, ainsi que l'intensité et la rapidité des changements socio-économiques, communicationnels et techniques propres aux sociétés occidentales et au processus de mondialisation, entraînent des disparitions en tout genres: de personnes et de collectivités humaines; de modes de vie, de production, de communication; d'objets rendus obsolètes ou mis hors circulation; d'espèces, de paysages, d'écosystèmes entiers. Dans tous ces cas, ce qui disparaît persiste dans son absence, de manière irrésolue, certes, mais incitant aussi l'émergence des nouvelles modalités de présence ou de vie.

Nous parlerons de disparition pour autant qu'il y ait suspension ou interruption plutôt que clôture ou accomplissement et qu'il manque la certitude de la fin qui aurait permis d'identifier et articuler une finalité. Ce qui est ainsi suspendu serait la capacité d'une chose ou personne quelconque de se manifester à la conscience humaine en tant que phénomène - objet d'expérience possible impliquant la liaison entre données des sens, situées dans le temps et dans l'espace, et catégories de pensée. S'ensuit presque inévitablement une problématique d'ordre ontologique, déjà présente, sinon dans l'étymologie latine du mot « disparaître» (pareo), du moins dans la définition ambiguë que le dictionnaire français nous en propose : ne plus être vu ou visible, donc devenir introuvable ou, plus simplement, s'en aller; mais aussi cesser d'être ou d'exister. La combinaison des deux sens nous rappelle la vieille question: dans quelle mesure différents degrés et manières de se manifester équivalent à différents modes et genres d'existence et aux tensions entre émergence et obscurcissement, repli et dévoilement, qui en seraient corrélatives? Le renversement opéré par rapport à la perspective usuelle de la quête phénoménologique n'est d'ailleurs pas sans importance: face à une disparition, ne serait-ce que celle dans un jeu de prestidigitation ou d'un tour d'illusionnisme, on n'interroge pas le phénomène mais son effacement et absence, tout en postulant la persistance éventuelle de la chose qui aurait disparu. De surcroît, on interroge de telles absences en des termes qui confinent aux impasses de la métaphysique et problématisent les outils conventionnels d'observation concrète, de connaissance positive et de communication discursive. L'idée et l'événement de la disparition mettent ainsi en relief la tournure aporétique qui marque toute problématique concernant les relations entre être et paraître.

Lorsque la disparition acquiert, comme nous croyons que c'est le cas dans notre époque, une dimension paradigmatique, elle affecte en tant que figure de pensée, notre manière de comprendre, de représenter et de faire l'expérience 
de la transformation et la constance, le changement et le devenir dans le temps et l'espace. Dans la mesure où ce qui disparaît n'est pas anéanti, il est possible d'associer au disparaître des modes particuliers d'existence soutenue ou de survie. La disparition fonctionne même, selon les contextes politiques et économiques, comme la figure qui cache des modes de persistance et signale aussi les conversions, déplacements et différentes opérations de matérialisation (ou d'immatérialisation) qui les maintiennent en vigueur. Pour ce qui est de l'observateur, ces modes d'existence sont toujours marqués, de manière plus ou moins aiguë et problématique, par le registre de l'incertitude - du possible, de l'éventuel, du probable. C'est ainsi que la disparition affecte toute forme de constitution et de transmission de connaissance, d'évaluation ou de critique et de mémoire de ce qui a ou qui aura eu lieu, en modifiant la matière même de ce dont il s'agit, devenue en quelque sorte informe, sans résolution, crucialement ouverte sur le temps et l'espace. C'est pourquoi, qu'elle soit perçue comme une donnée insaisissable ou comme un fait accompli, comme une libération ou comme une menace, la disparition met radicalement en question, non seulement les récits, mais bien les expériences et les structures de perception et modèles de représentation que ceux-ci devaient transmettre. Fin, forme, continuité, temporalité linéaire, ordre de séquences événementielles, projection dans l'avenir, l'idée même des personnes, événements ou objets jadis «sensibles»: tout doit être revu lorsque la langue ou tout autre moyen de configuration, de représentation, de transmission de matériaux ou de figures de vie se confrontent au disparaître, et aux modalités qu'il convoque.

La question de la disparition touche en effet de manière singulière celle des médias, du passage d'un médium à l'autre, des relations ou lacunes entre eux, pour autant que la production ou la reproduction de matériel ou de discours relevant de ce qui disparaît et de sa persistance, ou mémoire, soit entreprise par différents types de médias (oralité, écriture, image...) ou différents types de discours médiatiques (documentaire, fiction, poésie, mythe...). La quête à propos de ce qui disparaît nous confronte aux modes d'existence liminale qui semblent caractériser l'expérience contemporaine du réel, tissé par des liens et des creux intermédiaux - ces entre-deux où se jouent des tensions multiples non seulement de transformations, mais aussi de trans-matérialisations. Ces jeux et ces tensions entre présence et absence, disparition et réapparition, se déroulant dans et par la multiplicité et la variance actuelle de médias, font que l'idée ou le sentiment d'une substance ou même d'une existence certaine et compacte ou homogène deviennent particulièrement problématiques, et ce d'autant plus que ce qui disparaît le plus ou le plus crucialement, est, justement, l'entité matérielle par rapport à laquelle les signes médiatiques acquièrent le statut de « représentations ». 
Voilà donc quelques perspectives sur la notion de disparaître que nous avons essayé de combiner et de développer dans le présent numéro de la revue Intermédialités. Nous avons souhaité y inclure, pour ce faire, des contributions qui couvrent des domaines de recherche assez divergents dans le but d'explorer, de manière indicative, certes, et sans aucune prétention d'exhaustivité ou de globalité, un certain nombre d'incidences variées du problème de la disparition tant sur les catégories qui organisent la pensée et la connaissance que sur les manières contemporaine de vivre et de reconstruire l'expérience des relations humaines et de leur histoire.

Les articles de Valeria Wagner et de Sylvano Santini, ainsi que celui de Nicolas Zufferey, situent la notion de la disparition dans le cadre de différentes traditions philosophiques. C'est la perspective épistémologique qui prime dans le cas du rapprochement comparatif que Wagner opère entre Marx et Wittgenstein, pour interroger les manières dont leur discours théoriques impliquent une critique de la disparition que subissent les relations ou pratiques humaines sous le poids de conceptions sociales ou de conceptualisations linguistiques qui tendent à en effacer la portée. De son côté, Santini interroge systématiquement les relations de la pensée poétique, notamment mallarméenne, et philosophique, notamment bergsonienne, du point de vue de la manière dont elles relient la notion de la disparition avec les changements qui marquent, à leur époque, la conception occidentale du langage et de la technique, voire, plus généralement, de la médiation. C'est ce cadre de la culture occidentale que Zufferey met en relief, en le comparant, à travers des exemples tirés de la tradition chinoise, à une autre manière de concevoir les relations entre l'idée de la disparition et la question ontologique.

Différentes configurations du problème de la disparition dans le champ de la littérature et des arts constituent l'objet des articles d'Alain Brossat, Susan Bruce, Jean-Louis Déotte, David Guimond et Corinne Fassbinden. A travers l'exemple de la nouvelle Le Colonel Chabert, Brossat analyse l'histoire d'un disparu qui revient de la guerre et épuise tous les moyens pour faire reconnaître son droit à exister contre les mécanismes d'occultation et d'anéantissement mis en place par le système juridique - histoire avec laquelle Balzac aurait contribué «à l'histoire des vaincus», qui serait toujours, en un sens, « une histoire de la disparition». La difficulté de reconnaître et de gérer les implications de la disparition est aussi en jeu dans le cas des textes littéraires que Bruce analyse. Il est question, ici, de la disparition de personnages de fiction, qui peut ouvrir sur des questions d'éthique et de responsabilité pour autant que les lecteurs (ou le public) interpellés interrogent l'économie narrative et sa politique au-delà de la question narrative 
du sort des personnages. Ce type de lacunes textuelles évoque les limites du discours narratif quant à la représentation d'un événement qui, selon Déotte, est à la fois un non-événement, et un «archi-événement». Résistant à tout enchâ̂nement temporel et précédant «tout événement possible», la disparition entrave la séquence narrative, puisqu'elle marque une événementialité qui ne peut être saisie ou «accueillie» sous forme d'événement. Ce serait alors l'appareil vidéo, l'image vidéo, qui serait la plus apte à capter l'événement du disparaître, tout en protégeant vidéaste et spectateur de la suspension temporelle et spatiale qui lui est corrélative. Nous retrouvons un écho de ces propos dans les réflexions de Guimond sur les «sons", dont la disparition s'avère être un aspect constitutif d'un flux musical se déroulant à travers des apparitions et disparitions sonores simultanées. Mais ici, au lieu de suspendre le temps et de placer l'auditeur entre les moments inconcevables de l'avant et de l'après de la disparition, la musique devient plutôt le médium qui permet, par sa matérialité tenue et spécifique, que l'événement et le non-événement se confondent en déconstruisant la distinction visuelle entre l'apparaître et le disparaître. Dans le cas de l'artiste présenté dans l'article de Fassbinden, nous avons une modalité assez singulière du rapport d'autorité qui lie la présence de l'auteur à celle de ses créations, puisque c'est en disparaissant sous sa fausse identité que l'artiste en question confère de l'originalité à ses œuvres d'escroquerie et met en avant ses stratégies de fraude artistique. Ce faisant, le support économique, immatériel et discursif de la production artistique, longtemps occulté par la matérialité de l'œuvre et par son soutien autorial, est aussi mis de l'avant de manière incontournable.

Les articles d'Anita Starosta et de George Varsos portent sur des cas dans lesquels la question de la disparition se pose au sujet d'entités théoriquement problématiques d'ordre social ou institutionnel, interrogeant les présupposés et le modèles qui leur servent de fondement. Dans sa discussion du phénomène, autant matériel que conceptuel ou culturel, de la disparition de l'Union soviétique, Starosta analyse, à travers la «lunette» de la notion de la disparition, les modalités de changement historique que les modèles historiques en cours ne permettent pas de discerner, identifiant, d'une part, les médiations, et de l'autre, les tropes et les discours qui les occultent. Varsos interroge, de sa part, les implications éventuelles, pour l'écriture de la traduction littéraire, de la disparition de la langue de l'original, ce qui nécessite l'examen critique des présupposés théoriques qui postulent des relations étroites entre langues nationales et cultures historiques. Ainsi la figure de la disparition, pour autant que ses implications soient confrontées, acquiert encore une fois de la force critique. 
En clôture de notre numéro, Maria-Thalia Carras nous présente l'œuvre de Maria Papadimitriou: dans des lieux où les schèmes et les pratiques des conventions sociales dominantes disparaissent ou ont déjà disparu, l'artiste trace des voies et des figures qui permettent de percevoir aussi bien l'émergence de relations inédites que l'éruption de souvenirs persistants. Son œuvre met ainsi en relief un point sur lequel tous les articles de ce numéro convergent, chacun à leur manière; à savoir, qu'au-delà de sa fonction déconstructive, qui révèle les liens problématiques entre les structures d'expérience, les catégories de la pensée et de la représentation ainsi que les médias qui les déploient, la figure de la disparition a aussi une fonction productive, en ce qu'elle laisse entrevoir des expériences et des critères de perception inattendus. 